\title{
Démissions et licenciements face aux changements dans les entreprises :
}

La diversité des transitions professionnelles

Resignations and dismissals in a context of organizational changes: the diversity

of professional transitions

\section{Fabienne Berton}

\section{OpenEdition}

Journals

Édition électronique

URL : https://journals.openedition.org/travailemploi/6142

DOI : 10.4000/travailemploi.6142

ISSN : $1775-416 \mathrm{X}$

\section{Éditeur}

DARES - Ministère du Travail

\section{Édition imprimée}

Date de publication : 14 décembre 2013

Pagination : 49-68

ISSN : 0224-4365

\section{Référence électronique}

Fabienne Berton, «Démissions et licenciements face aux changements dans les entreprises : », Travail et Emploi [En ligne], 136 | octobre-décembre 2013, mis en ligne le 01 octobre 2015, consulté le 30 juillet 2021. URL : http://journals.openedition.org/travailemploi/6142 ; DOI : https://doi.org/10.4000/ travailemploi.6142 


\title{
Démissions et licenciements face aux changements dans les entreprises: la diversité des transitions professionnelles
}

\author{
Fabienne Berton $\left.{ }^{*}\right)$
}

Quelles sont les transitions professionnelles des salariés dont le contrat de travail est volontairement ou involontairement rompu dans un contexte de changements organisationnels et technologiques de leur entreprise? L'enquête COI 2006 et sa post-enquête qualitative réalisée deux ans plus tard permettent d'analyser ces transitions dans une problématique qui articule déterminants structurels et stratégies individuelles. Cinq types de transition professionnelle sont mis au jour dans lesquels jouent, de façon différenciée, la manière dont le salarié vit le changement, la pérennité de son emploi, l'initiative de la rupture de son contrat de travail et, pour terminer, sa capacité à mobiliser un projet professionnel. Entre les transitions choisies qui prennent la forme d'une démission et qui s'ouvrent sur une trajectoire favorable pour le salarié et les transitions subies qui se traduisent par un licenciement et où le risque d'exclusion est fort, il existe une zone intermédiaire faite aussi bien de démissions que de licenciements, de choix que de contraintes, dont cet article dessine quelques contours.

Face aux changements, et particulièrement aux réorganisations internes, que connaissent de façon plus ou moins permanente les entreprises, comment les salariés réagissent-ils? Ces réorganisations sontelles un facteur de rupture volontaire ou involontaire de leur contrat de travail lorsqu'il est à durée indéterminée? Quelles sont les transitions professionnelles que l'on observe à cette occasion? Quels en sont les déterminants principaux? Quelle place prennent les choix individuels par rapport aux contraintes structurelles? De nombreux travaux traitent des effets des plans sociaux résultant de restructurations financières ou de fusions-acquisitions, mais il est moins fréquent de poser la question de façon plus générale. Le travail mené ici ne s'intéresse pas aux arbitrages ni aux décisions des entreprises mais aux motivations et aux actions des salariés : quel rôle joue un environnement de travail mouvant sur la période qui entoure l'événement que constitue la rupture de contrat de travail, que cette rupture ait été volontaire ou non?

La post-enquête Changements organisationnels et informatisation 2006 (COI) permet d'approfondir cette question (voir encadré 1). Une trentaine d'entretiens semi-directifs a été réalisée entre le troisième trimestre 2008 et le premier trimestre 2009 auprès d'un échantillon aléatoire de salariés qui avaient répondu au volet salarié de l'enquête COI de 2006. Menés dans le cadre d'une convention Dares ${ }^{(1)}$-Cnam,

(*) Laboratoire interdisciplinaire pour la sociologie économique (Lise)-CNRS, Conservatoire national des arts et métiers (Cnam); fabienne.berton@cnam.fr.

(1) Dares: Direction de l'animation de la recherche, des études et des statistiques. ces entretiens avaient pour finalité de replacer la rupture du contrat de travail au sein de la trajectoire professionnelle des salariés qui étaient en outre plus particulièrement questionnés sur leur interprétation des événements immédiatement précédents et immédiatement suivants (BERTON, Perez, 2010).

\section{Encadré 1}

\section{L'enquête Changements organisationnels et informatisation 2006 (CO)}

Cette enquête (1) a pour objectif de connaître les changements organisationnels et technologiques opérés par les entreprises françaises et leurs effets sur le travail et les travailleurs entre 2003 et 2006. Un échantillon représentatif des entreprises françaises de dix salariés et plus du secteur marchand a été interrogé et un tirage aléatoire de quelques salariés par entreprise a été réalisé. Le fichier des "salariés ayant quitté l'entreprise " correspond aux salariés des entreprises $\mathrm{COI}$ qui, à la date où ils ont été interrogés, n'étaient plus dans l'entreprise pour laquelle ils ont été tirés au sort. Ce dernier fichier comprend 1394 salariés (fins de contrats à durée déterminée [CDD], démissions, licenciements, départs en retraite, congés, reprises d'études, mutations, etc.). Parmi eux, une trentaine de salariés qui ont démissionné ou ont été licenciés, a été retrouvée et a participé à un entretien deux ans plus tard.

(1) Pour plus de détails, voir le site : http://www.enquetecoi.net/; consulté le 30 novembre 2013 
Le droit du travail français différencie en 2006 deux grandes modalités de rupture du contrat de travail : l'une où la décision revient au salarié, la démission; l'autre où la décision provient de l'employeur, le licenciement ${ }^{(2)}$. Ce dernier est provoqué soit pour motif personnel (LMP) si l'employeur juge que le salarié a commis une faute, fait preuve d'insuffisance professionnelle ou souffre d'une inaptitude physique, soit pour motif économique (LME) dans les cas où l'entreprise fait face à des difficultés économiques sérieuses, à des mutations technologiques, à la nécessité de sauvegarder sa compétitivité ou doit cesser son activité. L'origine de la rupture est juridiquement claire. Mais qu'en est-il dans la pratique? N'existe-t-il pas des cas où la démission est imposée au salarié et d'autres où le salarié demande à être licencié? De telles questions conduisent à avancer l'hypothèse que la forme juridique de la rupture est peut-être moins importante que les conditions qui l'ont entourée.

Axé sur l'exploitation de données recueillies auprès de salariés, cet article expose dans une première partie la problématique suivie, qui est centrée sur les transitions et les ruptures professionnelles dans les trajectoires des salariés, considérées comme un lieu d'interaction entre déterminants structurels et stratégies individuelles. Dans une seconde partie, quelques statistiques tirées de l'enquête COI 2006 montrent que les populations de salariés se distinguent fortement selon leur situation à la date de l'enquête : toujours dans l'entreprise, démissionnaires, licenciés pour motif personnel ou pour motif économique. Une troisième partie rend compte de l'analyse des entretiens réalisés qui, dans une approche biographique, met en évidence cinq types de transition professionnelle dans lesquels jouent de façon différenciée plusieurs facteurs : la manière dont le salarié vit le changement dans son entreprise, la pérennité de son emploi, l'initiative de la rupture de son contrat de travail et, enfin, sa capacité à mobiliser un projet professionnel. Ces cinq types de transition, qui vont des plus favorables aux plus difficiles, sont présentés de façon plus approfondie dans une dernière partie.

\section{Transitions et ruptures professionnelles}

Ce travail sur les démissions et les licenciements dans un contexte de changement organisationnel et technologique des entreprises s'inscrit dans le prolongement des travaux portant sur les trajectoires professionnelles des individus, dans une problématique attentive à la fois à leurs déterminants structurels, tels que les modes de gestion de l'emploi

(2) En 2006 la rupture conventionnelle de contrat de travail à durée indéterminée n'existait pas. des entreprises, et à la façon dont les individus suivent différentes logiques d'action. «Les trajectoires des individus sur le marché du travail sont un révélateur du fonctionnement de ce marché. Elles renseignent à la fois sur les modalités de gestion de la main-d'œuvre des entreprises et sur les stratégies professionnelles que les individus développent pour s'adapter aux conditions qui s'imposent à eux" (BERTON, 2000, p. 356). Les stratégies individuelles ne sont pas seulement un calcul réalisé à chaque instant selon une rationalité en finalité ou en valeur. Elles expriment aussi l'épaisseur individuelle à travers ses différents espaces de socialisation : «Les acteurs sociaux concrets déploient des stratégies qui renvoient à des intérêts mais aussi à des valeurs; à des objectifs économiques mais aussi à des affinités culturelles; à des ressources stratégiques, mais aussi à des parcours biographiques » (DUBAR, 2001, p. 34).

Prêter plus particulièrement attention aux transitions, c'est-à-dire aux passages entre les séquences dont sont constituées les trajectoires, permet de pointer une des principales transformations dans la forme des trajectoires actuelles. Depuis les années 1980, pour toutes les catégories de salariés, on observe un même phénomène qui recouvre des situations différentes : l'accroissement du nombre moyen d'interruptions de carrière (BERTON et al., 2011, p. 14). Les travaux sur les transitions professionnelles se développent dans la littérature selon une dualité entre, d'un côté, des travaux qui s'intéressent avant tout à leurs déterminants structurels et, d'un autre, ceux qui mettent en avant les stratégies individuelles, contraintes ou non contraintes, des salariés.

Du côté des déterminants structurels des transitions professionnelles, se trouvent les travaux qui relient mobilité professionnelle et théories de la segmentation du marché du travail. Sont représentatifs de ces approches, par exemple, les recherches de Thomas Amossé et ses coauteurs (2011) qui ont pour objectif de «discuter la différenciation des trajectoires individuelles en termes de segmentation du marché du travail» (p. 81) et celles de Mireille BRUYÈrE et Laurence LizÉ (2010) qui se proposent d'analyser «dans quelle mesure les mobilités sur le marché du travail sont structurées et différenciées par les modes de gestion de l'emploi des entreprises» (p. 96). Ces recherches réactivent dans une perspective dynamique les travaux anciens sur les modes sectoriels de gestion de la main-d'œuvre des entreprises, initiés en 1982 pour les secteurs industriels (Blosseville et al., 1982). Elles mettent au cœur du fonctionnement du marché du travail les décisions d'emploi des entreprises (recrutements, formation, structuration des emplois, organisation du travail, gestion des carrières). Les travaux des années 1980 identifiaient statiquement les secteurs d'activité selon leur caractère plus ou moins stabilisateur de la main-d'œuvre en opposant les secteurs 
à gestion interne et les secteurs à gestion externe et, parmi ces derniers, ceux qui servaient de tremplin à l'insertion professionnelle des jeunes. Dans le contexte actuel, les différentes formes de flexibilité du travail développées par les entreprises valorisent la mobilité et l'adaptation permanente des salariés. Les recherches sur la flexibilité externe se sont d'abord attachées à souligner le développement des formes particulières d'emploi, telles que les CDD, l'intérim, le travail à temps partiel et les relations de sous-traitance. Dans un premier temps, elles se sont peu penchées sur la flexibilité qui touche les relations d'emploi permanentes telles que le CDI. Ce n'est qu'à l'occasion de la remise au jour des théories de la segmentation du marché du travail dans les années 2000 avec la thèse d'Héloïse Petit (2002) que la réflexion s'est approfondie sur «la déstabilisation des salariés stables» (CASTEL, 1995, p. 409) au sein de marchés internes renouvelés et eux-mêmes segmentés. De nature essentiellement quantitative, ces travaux remettent peu en cause les liens entre mobilité choisie, démission et issue favorable, et entre mobilité subie, licenciement et risque de chômage, de déclassement ou d'exclusion.

D'autres travaux, plus sociologiques, mais qui viennent nourrir le constat du renouvellement $\mathrm{du}$ fonctionnement des marchés internes du travail, se sont intéressés à la transformation des normes du travail et de l'emploi en soulignant la multiplication, dans les grandes entreprises souvent multinationales, d'une gestion de la main-d'œuvre sans engagement où les postes de travail ne sont plus garantis aux salariés. Le rapport au travail et à l'emploi devient alors paradoxal avec, d'un côté, l'exigence de motivation au travail et d'adhésion à l'entreprise et, d'un autre, l'injonction à ne pas s'attacher. Dans une telle conception des choses, certaines interruptions professionnelles, comme le licenciement, sont déjà présentées dans les discours et les pratiques de gestion comme un élément courant, voire normal, des trajectoires d'emploi (GUYONVARCH, 2008, p. 149), occultant le fait qu'il s'agisse de ruptures souvent vécues sur le mode de l'épreuve. Toutefois, l'origine de l'interruption n'est pas à rechercher uniquement du côté des services de direction ou des ressources humaines. Le contexte professionnel dans lequel est plongé le salarié et en particulier la dégradation des conditions de travail liée à son intensification (Gollac, 2005) peuvent être un déterminant essentiel de la rupture. Cette question est difficile à appréhender. Les différents travaux qui cherchent à relier les changements organisationnels à des questions de conditions de travail (accident du travail, santé, bien-être au travail), débouchent sur une indécision : il est difficile d'imputer à un changement un effet clair sur la santé ou les conditions de travail des salariés concernés tant les situations varient selon les contextes de mise en œuvre, les caractéristiques des entreprises et celles des salariés. L'intensification ne peut pas être assimilée à une dégradation générale du travail ou du rapport au travail. Elle peut correspondre à un investissement de soi gratifié et gratifiant, tout en induisant un risque plus élevé de souffrance mentale (Gollac, 2005 , p. 212). En revanche, parmi les échecs des changements organisationnels, le facteur humain est un élément important à prendre en considération, ce que Monique Combes et Laëtitia LethielLeux (2008, p. 336) appellent la compréhension de la nature sociotechnique des organisations. Les changements d'organisation se heurtent souvent à des difficultés concernant le personnel, qu'il s'agisse de problèmes relatifs à l'adaptation au changement ou à l'utilisation des nouveaux outils, ou encore de problèmes de moyens. La formation du personnel, les délais et les moyens accordés apparaissent centraux pour la mise en œuvre des changements organisationnels (Kocoglu, Moatty, 2010, p. 225).

Du côté des stratégies individuelles, certains travaux récents ont analysé, dans une perspective biographique, les transitions professionnelles en termes de bifurcation et de rupture (BESSIN et al., 2010)(3). Les recherches françaises sur les ruptures professionnelles s'intéressent fréquemment aux reconversions professionnelles volontaires (Negroni, 2005; Denave, 2006) entendues comme «des changements d'activité, de secteur ou de profession opérés de manière volontaire » (NEGRONI, 2005, p. 313); elles ont également lieu «quand le métier exercé sollicite de nouvelles compétences, de nouveaux savoir-faire et s'effectue dans un domaine professionnel sans lien avec le précédant» (Denave, 2006, p. 86). Entrent en jeu dans ces ruptures professionnelles volontaires, non seulement l'anticipation et le caractère volontaire du changement d'activité, mais aussi le moment précis de survenue de l'événement: celui où le décalage avec l'activité précédente augmente l'insatisfaction professionnelle (Negroni, 2005; Denave, 2006). Parmi les moyens de réalisation de la rupture, le hasard peut jouer un rôle: "précisément parce qu'il s'inscrit dans une configuration particulière qui est celle dans laquelle se trouve l'individu à un instant précis. Il ne se déclenche que parce qu'il y a une direction préexistante en filigrane» (NEGRONI, 2005 , p. 317). Outre le hasard, la médiation peut aussi prendre la forme d'une personne physique. Catherine Negroni (2005, p. 324) utilise le terme de «passeur» pour désigner la tierce personne grâce à laquelle la bifurcation se réalise. Un autre type de rupture professionnelle, contrainte cette fois, est analysée par Cécile Vignal (2005a, 2005b, 2006) dans le cas précis de la délocalisation d'une usine qui oblige les ouvriers à «choisir» entre un déménagement, une double résidence ou la recherche d'un autre emploi. Dans ce cas, ainsi que le montre Vignal (2005b, p. 182), la prise en compte du

(3) Dans la poursuite des travaux sur la sociologie des bifurcations (GrossetTI, 2006). 
facteur familial permet de comprendre pourquoi les salariés souhaitent une stabilité résidentielle qui ne résulte pas uniquement d'impératifs professionnels et économiques.

\section{Quelques données de cadrage issues des fichiers couplés de l'enquête COI 2006}

Les statistiques descriptives de l'enquête couplée salarié/employeur COI 2006 apportent quelques éclairages sur les ruptures de contrat de travail. Les populations de salariés y apparaissent nettement différenciées (voir tableaux 1 et 2).

Tableau 1 : Statistiques descriptives des salariés en 2006 (en \% de salariés ou en nombre d'années)

\begin{tabular}{|c|c|c|c|c|}
\hline Caractéristiques des salariés & 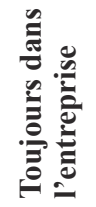 & 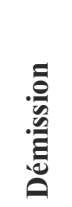 & $\sum^{\hat{S}}$ & $\sum_{-1}$ \\
\hline Femmes & 37,5 & 34,1 & 45,8 & 39,5 \\
\hline Plus de 40 ans & 50,5 & 22,5 & 40,8 & 50,3 \\
\hline Âge moyen (en années) & 40 & 33 & 38 & 39 \\
\hline Diplôme supérieur à bac +2 & 15,4 & 21,8 & 17,9 & 15,1 \\
\hline Ouvriers & 39,8 & 34,1 & 34,1 & 43,2 \\
\hline $\begin{array}{l}\text { Plus de } 5 \text { ans d'ancienneté dans } \\
\text { l'entreprise }\end{array}$ & 75,9 & 43,5 & 49,7 & 69,2 \\
\hline Ancienneté moyenne (en années) & 13 & 5 & 9 & 11 \\
\hline $\begin{array}{l}\text { Charge de famille supérieure ou } \\
\text { égale à un }\end{array}$ & 58,9 & 46,5 & 44,7 & 57,8 \\
\hline$N$ & 14369 & 454 & 179 & 185 \\
\hline
\end{tabular}

Lecture : LMP : licenciement pour motif personnel; LME : licenciement pour motif économique.

Champ : Salariés des entreprises françaises de dix salariés et plus du secteur marchand.

Source : COI 2006, traitement de l'auteur.

Ceux qui restent dans leur entreprise en 2006 sont plus âgés, ils ont une ancienneté dans l'emploi supérieure et ont plus souvent charges de famille que ceux qui démissionnent ou sont licenciés. Ce résultat est cohérent avec un rôle protecteur de l'ancienneté dans l'emploi, non seulement vis-à-vis du licenciement mais aussi vis-à-vis de la démission, déjà souligné par la littérature : «Les grandes entreprises, où ont été largement observés les marchés internes et leurs contrats de long terme, ont particulièrement protégé leurs salariés anciens d'âge médian» (BeHaGHeL, 2003, p. 21). Appartiennentils à un marché interne? La réponse est oui, si on considère en même temps les caractéristiques de leur entreprise en termes d'effectifs salariés et de secteur d'activité. Ils travaillent plus fréquemment dans des entreprises de plus grande taille et dans des secteurs réputés développer la gestion interne de leur main-d'œuvre : activités financières, immobilières, énergie, etc.
Tableau 2 : Statistiques descriptives de leurs entreprises à la même date (en \% de salariés)

\begin{tabular}{|c|c|c|c|c|}
\hline & 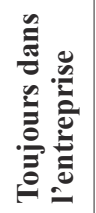 & 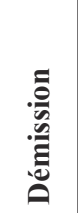 & $\sum^{O}$ & 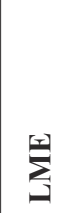 \\
\hline \multicolumn{5}{|l|}{ Taille de l'entreprise } \\
\hline Moins de 50 salariés & 19,9 & 31,7 & 31,8 & 22,2 \\
\hline De 50 à 199 salariés & 19,7 & 21,6 & 24,1 & 33,5 \\
\hline De 200 à 499 salariés & 15,4 & 11,5 & 13,4 & 15,1 \\
\hline 500 salariés et plus & 46 & 35,2 & 30,7 & 29,2 \\
\hline \multicolumn{5}{|l|}{ Secteur d'activité } \\
\hline $\begin{array}{l}\text { Industries agricoles et } \\
\text { alimentaires }\end{array}$ & 6,8 & 5,7 & 6,2 & 5,4 \\
\hline $\begin{array}{l}\text { Industries des biens de } \\
\text { consommation }\end{array}$ & 6,3 & 3,5 & 6,2 & 10,3 \\
\hline Industrie automobile & 2 & 0,7 & 0,6 & 1,1 \\
\hline Industries des biens d'équipement & 8 & 3,1 & 2,8 & 6,5 \\
\hline Industries des biens intermédiaires & 15,6 & 6,2 & 7,8 & 22,7 \\
\hline Énergie & 1,3 & 0,7 & 0,6 & 0,5 \\
\hline Construction & 6,4 & 6,4 & 7,3 & 2,7 \\
\hline Commerce et réparations & 17,7 & 26,9 & 25,1 & 15,1 \\
\hline Transports & 7,4 & 8,6 & 7,3 & 11,4 \\
\hline Activités financières & 6,4 & 2,2 & 3,4 & 1,1 \\
\hline Activités immobilières & 1,7 & 1,3 & 0,6 & 0 \\
\hline Service aux entreprises & 16,9 & 25,6 & 23,5 & 21,1 \\
\hline Service aux particuliers & 3,8 & $\mathbf{9 , 3}$ & 8,9 & 2,2 \\
\hline \multicolumn{5}{|c|}{ Changements dans l'entreprise depuis 3 ans } \\
\hline Un changement important & 44,1 & 37,7 & 43 & 44,3 \\
\hline Une restructuration financière & 33,4 & 32,6 & 32,4 & 40 \\
\hline Une refonte de l'organigramme & 49,1 & 42,3 & 51,4 & 58,4 \\
\hline $\begin{array}{l}\text { Une restructuration financière et } \\
\text { une refonte de l'organigramme }\end{array}$ & 25 & 22,5 & 26,3 & 33 \\
\hline Une délocalisation & 8 & 3,7 & 3,9 & 14,6 \\
\hline $\begin{array}{l}\text { Une implantation de site à } \\
\text { l'étranger }\end{array}$ & 16,7 & 13,4 & 8,4 & 10,8 \\
\hline $\begin{array}{l}\text { Plus de } 3 \text { changements } \\
\text { d'organisation }\end{array}$ & 8,8 & 6,6 & 3,9 & 10,8 \\
\hline$N$ & 14369 & 454 & 179 & 185 \\
\hline
\end{tabular}

Lecture : LMP : licenciement pour motif personnel; LME : licenciement pour motif économique.

Champ : Salariés des entreprises françaises de dix salariés et plus du secteur marchand.

Source : COI 2006, traitement de l'auteur.

Ceux qui démissionnent sont plus fréquemment des hommes, plus jeunes, plus diplômés, avec moins d'ancienneté dans l'entreprise et moins de charges de famille que le reste de la population. Ils travaillent davantage dans des entreprises de petite taille (moins de cinquante salariés), dans des secteurs du commerce et des services connus pour leur gestion de la main-d'œuvre marquée par la flexibilité externe. Mais un certain nombre d'entre eux provient aussi d'entreprises de grande taille, ce qui suggère l'existence de deux populations au sein des démissionnaires : des salariés insérés dans des secteurs à flexibilité externe d'une part, et des jeunes en phase d'ascension professionnelle et de recherche d'une entreprise où se stabiliser et mieux valoriser leurs compétences acquises d'autre part : 
«Qualification is acquired within the firm but is most frequently valued through external mobility» (Gazier, Petit, 2007, p. 1031). Quels qu'ils soient, ils sont associés à moins de changement dans leur entreprise que l'ensemble de la population, à l'exception des implantations de sites à l'étranger qui peuvent être interprétées comme un indicateur de bonne santé de l'entreprise.

Ceux qui sont licenciés pour motif personnel (LMP) sont plus fréquemment des femmes avec moins souvent une charge de famille que l'ensemble de la population. Les secteurs d'activité sont également ceux connus pour leur gestion de la main-d'œuvre marquée par la flexibilité externe : construction, commerce et services, au sein d'entreprises qui sont de taille petite ou moyenne. La principale modalité de changement qui leur est associée est la réorganisation interne (refonte de l'organigramme), qui invite à formuler l'hypothèse d'un recours privilégié à ce type de licenciement dans le cas d'entreprises en réorganisation dans ces secteurs (BEAUJOLIN et al., 2012, p. 8) ${ }^{(4)}$.

Ceux qui sont licenciés pour motif économique (LME) le sont avant tout dans un contexte de restructuration financière, de refonte de l'organigramme et de délocalisation qui concernent des entreprises de taille moyenne (entre 50 et 200 salariés), des secteurs des industries de biens de consommation, de biens intermédiaires et des transports. Ce sont plus fréquemment des ouvriers, plus âgés, d'un moindre niveau d'éducation et avec charges de famille.

Ces statistiques n'apportent pas véritablement de connaissances nouvelles sur les ruptures de contrat de travail. Elles confirment ce qui est maintenant bien connu des modes de gestion sectoriels de la main-d'œuvre et des profils de salariés licenciés et démissionnaires, et semblent a priori venir à l'encontre de notre hypothèse de travail en renforçant l'idée de pratiques d'entreprises et de population de salariés bien différenciées entre les différentes modalités juridiques de rupture du contrat de travail. Mais qu'en est-il d'une démission ou d'un LMP dans un contexte de restructuration financière et de délocalisation? Qu'en est-il des LME? Sont-ils tous associés à des restructurations financières?

Notre ambition est d'articuler déterminants structurels et stratégies individuelles dans l'analyse des transitions professionnelles occasionnées par une rupture de contrat de travail dans un contexte de changement organisationnel et technologique

(4) Ce résultat entre en résonnance avec d'autres travaux. À partir d'enquêtes de terrain, Florence PALPACUER et ses coauteurs (2007) montrent que, dans des contextes de restructurations financières, les cadres de certaines grandes entreprises sont licenciés pour motif personnel plutôt que pour motif économique. Tout récemment, Camille Signoretto et Julie Valentin (2012) montrent que les LMP sont nettement corrélés aux modalités de gestion de la main-d'œuvre des entreprises tandis que les LME le sont davantage à leur activité économique. des entreprises. Pour cela, il faut considérer que la rupture du contrat de travail n'est pas un événement instantané, ni isolé, même s'il peut apparaître plus ou moins rapidement et peut sembler plus ou moins brutal. C'est une transition plus ou moins prévisible, plus ou moins longue qui introduit en tout cas une irréversibilité : il n'est plus possible au salarié de conserver le même emploi. Les entretiens qualitatifs réalisés dans le cadre de la post-enquête COI 2006 deux ans plus tard ont été exploités dans une perspective biographique : le travail qualitatif prend en compte l'ensemble de la trajectoire professionnelle du salarié pour analyser sa transition professionnelle. Cette trajectoire est évaluée à partir de la situation du salarié deux ans après son départ et c'est à cette occasion que sont réinterprétés le contexte et les conditions de son départ de 2006. Le qualitatif permet à la fois la mise à jour des stratégies individuelles et des déterminants structurels complétés et approfondis.

\section{Les transitions professionnelles des salariés}

L'échantillon des 33 salariés rencontrés est issu des salariés qui ont répondu à l'enquête $C O I$ en 2006 et qui, à cette date, avaient démissionné ou avaient été licenciés. Il n'a aucun caractère représentatif. Sa constitution repose sur deux conditions : retrouver le salarié deux ans après la rupture de son contrat de travail et obtenir qu'il accepte un entretien d'une heure à une heure trente. De fait, par rapport à l'ensemble des salariés qui ont connu une rupture de leur contrat de travail dans l'enquête $C O I$ 2006, les salariés rencontrés dans la post-enquête se caractérisent par un niveau de diplôme nettement plus élevé : $54 \%$ ont un niveau de diplôme supérieur à bac +2 alors qu'ils ne sont que $19 \%$ dans l'enquête COI 2006. Ils ont donc plus fréquemment le statut de cadre : $43 \%$ contre $16 \%$.

L'analyse porte sur les justifications que les acteurs eux-mêmes donnent à leurs actions. Dans une telle optique, ces justifications ne sont pas exemptes de rationalisation ex post qui organisent le récit selon une logique significative. L'illusion biographique veut que le récit de vie se compose en "séquences ordonnées selon des relations intelligibles » selon le «postulat du sens de l'existence» (Bourdieu, 1986, p. 69). Cette justification ex post conduit, entre autres, à ce que la situation professionnelle antérieure à la rupture du contrat de travail soit systématiquement évaluée négativement de la part des salariés : "Lorsque la décision de changer d'emploi s'exprime dans la narration, la coupure est déjà faite avec l'univers professionnel qui est alors nécessairement appréhendé avec beaucoup de recul et de distance critique» (NEGRONI, 2005, p. 329). Une telle considération est à prendre au 
sérieux et nécessite de ne pas faire une transcription rudimentaire du discours du salarié, non pas qu'il s'agisse de le censurer ou de le contrôler mais de veiller à en exposer ensuite une interprétation correcte.

Adopter une perspective de trajectoire professionnelle, permet d'aller au-delà du moment de la rupture du contrat de travail afin d'analyser l'ensemble du processus de transition professionnelle. Plusieurs critères interagissent pour différencier les transitions professionnelles des salariés rencontrés : l'impact sur le salarié des changements organisationnels et technologiques vécus dans l'entreprise, la marge d'action dont le salarié dispose et, en particulier, la question de savoir si son emploi est supprimé et si l'initiative de la rupture du contrat de travail lui revient, enfin sa capacité à mobiliser un projet professionnel. Ce dernier peut être en réalité poursuivi de longue date et le salarié y renforce son ancrage à l'occasion de la rupture. Il peut s'agir d'un projet latent encore jamais réalisé mais alternatif aux emplois antérieurs. Ce peut être aussi une réorientation soudaine motivée par des circonstances telles que la saisie d'une opportunité d'emploi, la fermeture de l'établissement ou des conditions de travail dégradées par de mauvaises relations hiérarchiques, une organisation du travail instable ou des vagues de départ successives. Dans les cas où le salarié ne poursuit pas son activité dans le même domaine professionnel ou avec le même statut d'emploi, on parlera de rupture professionnelle, cette rupture professionnelle accompagnant la rupture de son contrat de travail. Ce sont ces critères qui vont permettre de catégoriser les transitions professionnelles de chacun des 33 salariés rencontrés.

\section{L'impact des changements organisationnels et technologiques vécus dans l'entreprise}

Une première question à se poser dans l'analyse des transitions professionnelles des salariés est celle de l'impact des changements organisationnels et technologiques vécus dans l'entreprise sur la rupture de leur contrat de travail. Soit des changements existent dans l'entreprise ou l'établissement mais le salarié ne s'estime pas touché; soit des changements existent et le salarié se juge directement touché; soit il y a peu de changement dans l'entreprise. Quels changements les salariés rencontrés restituent-ils?

Les restructurations des entreprises sont très diverses : rachats, fusions, scissions, filialisations, délocalisations, etc. Un contexte de restructuration peut servir de moteur au départ quand il vient augmenter l'insatisfaction professionnelle et faciliter le processus de désengagement de l'entreprise et de l'activité qui y est exercée. Les restructurations peuvent bousculer directement les parcours professionnels des salariés, soit que leur branche d'activité est supprimée dans l'entreprise, soit que leur emploi y devient redondant, soit que la gestion de la main-d'œuvre qui y est mise en œuvre vise à renouveler les équipes de travail(5). Parmi les salariés rencontrés, trois formes de restructurations sont observées (voir encadré 2).

\section{Encadré 2}

\section{Les trois formes de restructurations rencontrées dans la post-enquête}

Celles concernant de grandes unités, ou de plus petites appartenant à des groupes, insérées dans le capitalisme financier, qui se manifestent par des scissions, des fusions et des acquisitions. Quinze des salariés rencontrés sont dans cette situation: Bachir, Claire, Édouard, Étienne, François, Gabriel, Jean-Charles, Jonathan, Joseph, Laurent, Maurice, Pauline, Séverine, Sylvie et Yann. Certains salariés semblent ignorer ce contexte qui ne les atteint pas. D'autres remettent en cause l'organisation, voire la finalité, de l'entreprise. Sans aller jusqu'à une remise en question complète, d'autres encore prennent de la distance avec le travail subordonné et préfèrent investir des environnements de travail qu'ils jugent préférables (Claire, Édouard, Gabriel, Jean-Charles, Joseph, Sylvie).

Celles ensuite qui prennent la forme du rachat, par une plus grande unité, d'une petite ou d'une toute petite entreprise au moment de la retraite ou du décès de son dirigeant-créateur. Là aussi, certains salariés sont déstabilisés car ils sont souvent contraints à intégrer une unité bien plus grande que celle qu'ils quittent avec de tout autres méthodes, conditions de travail et relations avec leur direction, ce qui les pousse à en partir. Trois salariés rencontrés sont dans cette situation : Antonio, Christine et Myriam.

Celles enfin qui correspondent à la perte d'un marché pour des entreprises prestataires de services où les salariés sont dans une relation triangulaire d'emploi, en mission dans d'autres entreprises (Badia, Barbara, Lise). Dans ce cas, soit la convention collective de branche prévoit la mutation automatique du salarié et il n'y a pas de rupture du contrat de travail, soit rien n'est prévu (Lise).

Sans aller jusqu'aux restructurations financières, il est rare que les entreprises ne connaissent pas de changements organisationnels plus ou moins importants, ne serait-ce qu'un renouvellement hiérarchique (Adélaïde, Alia, Anne, Armand, Jean-Pierre, Mélanie, Oualid, Pierre,

(5) La pratique de gestion de la main-d'œuvre visant, lors de restructurations qui s'accompagnent d'un changement de direction, à renouveler les équipes de travail, est fréquente. Son but est de faciliter la transformation des attitudes, des valeurs et de la culture organisationnelle des salariés (HUDSON, 2002; DAYAN et al., 2008). 
Sophie, Victor). Il existe aussi des contextes de petites entreprises, où prévaut le modèle domestique décrit par l'économie des conventions. Le départ du salarié ne correspond pas tant à des changements d'organisation qu'à la dégradation des relations interpersonnelles face à un patron (ou un supérieur hiérarchique) tout puissant et autoritaire. Adélaïde, Sophie, Victor sont particulièrement représentatifs de ces situations. Il s'agit moins, dans leur cas, de réorganisation et d'introduction de nouvelles méthodes de travail que de perturbations du climat et des relations professionnels du fait de la personnalisation de la relation entre le salarié et la direction (ou le supérieur hiérarchique direct), qui non seulement détériorent les relations de travail mais peuvent aussi être interprétées comme une volonté hiérarchique de se séparer du salarié.

\section{La pérennité de l'emploi et l'initiative de la rupture du contrat de travail}

Un deuxième élément de clivage des transitions professionnelles observées est le fait de savoir si l'emploi que le salarié quitte en 2006 est un emploi supprimé dans l'entreprise. On pourrait penser, en première approche, que les démissions et les LMP correspondent à des emplois qui deviendront vacants dans l'entreprise après le départ du salarié et que seuls les LME correspondent à des réductions d'effectifs et donc à la suppression des emplois. La correspondance n'est en fait pas parfaite (voir les tableaux 1, 2, 3, 4 et 5 en annexe). Elle l'est pour les démissions qui sont toutes associées à des emplois non supprimés. En revanche pour les licenciements, les choses sont plus complexes : il existe des LME sans suppression de l'emploi du salarié (pour Myriam et Yann, il y a bien réduction globale de l'emploi dans leur entreprise, mais leur emploi précis n'est pas supprimé et ils partent volontairement dans le cadre d'un plan social); il existe des LMP avec suppression de l'emploi du salarié (Antonio, Catherine, Christine, Édouard, Joseph, Lise) lorsque l'employeur veut se séparer $\mathrm{du}$ salarié dans un contexte de réorganisation du travail, confirmant en cela l'usage privilégié des LMP par les entreprises dans le cas de réorganisations (cf. supra).

Le tableau 3 qui relie modalité juridique du départ, suppression ou non de l'emploi du salarié et initiative de son départ fait apparaître que les zones d'indécision portent sur les licenciements (LMP et LME) d'une part, en ce qui concerne la suppression ou non de l'emploi occupé par le salarié et d'autre part, en ce qui concerne l'initiative de son départ de l'entreprise.
Tableau 3 : Les correspondances entre modalité juridique de rupture du contrat de travail, initiative du départ et suppression de l'emploi du salarié

\begin{tabular}{|l|c|c|c|}
\cline { 2 - 4 } \multicolumn{1}{c|}{} & Démission & LMP & LME \\
\hline \multicolumn{1}{|c|}{ En principe sur le plan juridique } \\
\hline $\begin{array}{l}\text { Pérennité de } \\
\text { l'emploi }\end{array}$ & $\begin{array}{c}\text { Emploi non } \\
\text { supprimé }\end{array}$ & $\begin{array}{c}\text { Emploi non } \\
\text { supprimé }\end{array}$ & $\begin{array}{c}\text { Emploi } \\
\text { supprimé }\end{array}$ \\
\hline $\begin{array}{l}\text { Initiative de } \\
\text { la rupture }\end{array}$ & Départ choisi & Départ subi & Départ subi \\
\hline Situation réellement observée \\
\hline $\begin{array}{l}\text { Pérennité de } \\
\text { l'emploi }\end{array}$ & $\begin{array}{c}\text { Emploi non } \\
\text { supprimé }\end{array}$ & $\begin{array}{c}\text { Emploi supprimé ou non } \\
\text { supprimé }\end{array}$ \\
\hline $\begin{array}{l}\text { Initiative de } \\
\text { la rupture }\end{array}$ & Départ choisi & \multicolumn{2}{|c|}{ Départ subi ou choisi } \\
\hline
\end{tabular}

Champ : Salariés des entreprises françaises de dix salariés et plus du secteur marchand.

Source : Post-enquête COI 2006, traitement de l'auteur.

Cette question de la suppression de l'emploi du salarié est indissociable de celle de la marge d'action dont le salarié dispose et, en particulier, de celle de savoir si la décision de la rupture du contrat de travail en 2006 lui revient. L'initiative du départ peut aussi bien revenir au salarié lui-même qu'à son entreprise. En la matière, on ne peut s'en tenir à la forme juridique prise par la rupture (démission / licenciement) et à ce qu'elle sous-entend en termes d'origine de la décision. À cet égard, des travaux récents (Amossé et al., 2011, p. 80) montrent bien « une nette différenciation des profils de salariés selon que leur rupture d'emploi était subie, acceptée ou choisie» quand on s'attache à l'observation du devenir du salarié après la rupture. Si les démissions (au nombre de 16 dans notre post-enquête) sont bien toutes à l'initiative du salarié, tous les licenciements (14) ne sont pas symétriquement à l'initiative de l'employeur: certains salariés choisissent cette rupture de leur contrat de travail, soit en se portant volontaires pour intégrer un plan social dans le cadre d'un licenciement pour motif économique (Claire, Myriam, Yann), soit en demandant un licenciement car l'employeur ne fournit plus suffisamment de travail (comme dans le cas de Catherine, tout à fait particulier, présenté un peu plus bas). Poser l'alternative en termes d'initiative de la décision, plutôt qu'en termes strictement juridiques, permet en plus d'enrichir l'analyse par les marges de négociation que le salarié s'est données au moment de la rupture de son contrat de travail, sachant que ces marges jouent dans les deux sens, choisi comme subi.

Du côté choisi se trouvent tous les salariés qui ont été licenciés alors qu'ils ont pris l'initiative de leur départ (Catherine, Claire, Myriam, Yann). Du côté subi, tous les salariés qui ont réussi, souvent au prix d'un processus long et difficile, à transformer une démission imposée en un licenciement. C'est le cas d'Adélaïde, Antonio, Édouard et Joseph. Ce 
sont très clairement des cas où l'employeur veut se séparer du salarié qui, lui, ne souhaite pas partir(6).

Il est important de distinguer, parmi les négociations individuelles dans la période du départ du salarié, celles qui se déroulent dans un contexte négatif pour lui et celles qui se déroulent dans un contexte plus positif. Les contextes négatif s correspondent aux cas où l'employeur veut se séparer du salarié et le pousse à démissionner. Certains salariés réussissent à négocier une solution qui leur est plus avantageuse : un licenciement pour motif personnel qui ouvre des droits aux allocations-chômage. Pour un des enquêtés, l'employeur impose un licenciement pour faute professionnelle, le salarié accepte la faute et négocie des indemnités compensatrices (Caroline). D'autres négociations se déroulent dans un contexte plus favorable où, par exemple, le salarié s'inscrit volontairement dans un plan social en cours. Même si Rachel BeAujolin et ses coauteurs (2012) tiennent à souligner «l'ambiguiité du volontariat quand celui-ci s'inscrit dans un projet à l'initiative de l'employeur» (p. 9), le salarié bénéficie alors des négociations collectives qui s'appliquent à tous les salariés. Quelques-uns négocient en sus certains avantages à titre individuel : un départ immédiat sans préavis (Myriam), des indemnités plus élevées et une formation plus longue et plus coûteuse que celles prévues par le plan social (Yann). Hors plan social et procédures collectives de licenciement, d'autres salariés, qui prennent l'initiative de leur départ en démissionnant, peuvent négocier des conditions avantageuses telles qu'un congé sabbatique pour tester le nouvel emploi qu'ils ont en vue avant de poser leur démission (Anne); ils peuvent aussi choisir la date de leur départ de façon à obtenir un meilleur solde de tout compte (Mélanie). A contrario, il existe des cas où toute négociation est impossible : Pierre part sans effectuer son préavis avant même d'être certain d'avoir un nouvel emploi car, après avoir annoncé à son employeur son intention de démissionner, il est immédiatement mis à la porte. On est là dans une problématique de petite entreprise où dominent les relations interpersonnelles de nature affective. Heureusement, Pierre est technicocommercial en micro-informatique et l'embauche qu'il visait se concrétise rapidement. En règle générale, plus le salarié appartient à une catégorie socioprofessionnelle élevée, ce qui est très majoritairement le cas dans notre post-enquête, plus il s'autorise à négocier

(6) Selon l'enquête sur la formation et la qualification professionnelle (FQP) 2003 (Amossé et al., 2011, p. 95), «4\% des démissions sont considérées par les salariés comme à l'initiative de l'employeur avec leur accord... Elles peuvent renvoyer à des situations d'arrangement entre salariés et employeurs préfigurant les ruptures conventionnelles instaurées par la loi en $2008 »$. Autre situation d'arrangement préfigurant les ruptures conventionnelles rencontrées dans la post-enquête, le «protocole d'accord transactionnel de licenciement» d'Édouard (voir, plus bas dans l'article, la partie intitulée «Se reconvertir»). individuellement son départ et plus il obtient satisfaction (Joseph et Yann). Les ouvriers et employés, en revanche, prennent rarement ce type de libertés, les conditions de leur départ leur étant imposées.

\section{La capacité du salarié à mobiliser un projet professionnel}

La dernière dimension qui se dégage de l'analyse tient à la capacité ou non du salarié à mobiliser un projet professionnel. Nous pouvons distinguer cinq cas de figure (voir tableau 4 et encadré 3).

Tableau 4 : Différents types de transition professionnelle observés en fonction des critères présentés

\begin{tabular}{|l|c|c|c|}
\hline \multicolumn{1}{|c|}{$\begin{array}{c}\text { Type de } \\
\text { transition }\end{array}$} & $\begin{array}{c}\text { Poursuivre } \\
\text { sa carrière / } \\
\text { Trouver sa voie }\end{array}$ & $\begin{array}{c}\text { Se recon- } \\
\text { vertir / Se } \\
\text { réorienter }\end{array}$ & $\begin{array}{c}\text { Subir la } \\
\text { rupture }\end{array}$ \\
\hline $\begin{array}{l}\text { Impact du } \\
\text { changement* } \\
\text { sur le salarié }\end{array}$ & $\begin{array}{c}\text { Le salarié } \\
\text { n'y prête pas } \\
\text { attention }\end{array}$ & \multicolumn{2}{|c|}{$\begin{array}{c}\text { Le salarié s'estime } \\
\text { touché }\end{array}$} \\
\hline $\begin{array}{l}\text { Impact du } \\
\text { changement* } \\
\text { sur son emploi }\end{array}$ & $\begin{array}{c}\text { Emploi non } \\
\text { supprimé }\end{array}$ & $\begin{array}{c}\text { Emploi } \\
\text { supprimé } \\
\text { ou non } \\
\text { supprimé }\end{array}$ & $\begin{array}{c}\text { Emploi } \\
\text { supprimé }\end{array}$ \\
\hline $\begin{array}{l}\text { Modalité } \\
\text { juridique }\end{array}$ & Démission & $\begin{array}{c}\text { Démission, } \\
\text { LMP ou } \\
\text { LME }\end{array}$ & LMP \\
\hline $\begin{array}{l}\text { Initiative du } \\
\text { départ }\end{array}$ & Départ choisi & $\begin{array}{c}\text { Départ subi } \\
\text { ou choisi }\end{array}$ & $\begin{array}{c}\text { Départ } \\
\text { subi }\end{array}$ \\
\hline Projet \\
professionnel & Un projet & $\begin{array}{c}\text { Un projet } \\
\text { profes- } \\
\text { sionnel } \\
\text { latent ou } \\
\text { rapidement } \\
\text { mobilisé }\end{array}$ & $\begin{array}{c}\text { Pas de } \\
\text { projet } \\
\text { profes- } \\
\text { sionnel }\end{array}$ \\
\hline
\end{tabular}

* : les changements dans l'entreprise peuvent prendre la forme de rachat, fusion, restructuration financière, réorganisation, délocalisation, etc.

Champ : Salariés des entreprises françaises de dix salariés et plus du secteur marchand.

Source : Post-enquête COI 2006, traitement de l'auteur.

En synthèse, le tableau 4 fait découler les modalités juridiques de rupture du contrat de travail et les objectifs professionnels poursuivis par le salarié des critères présentés (impact des changements dans l'entreprise, pérennité de son emploi, initiative de son départ, capacité à mobiliser un projet professionnel). Les situations où le salarié ne prête pas attention aux changements dans son entreprise, où son emploi n'est pas supprimé et où il prend l'initiative de son départ, sont des démissions motivées par des objectifs de poursuivre sa carrière ou de trouver sa véritable voie. À l'opposé, les salariés qui s'estiment directement affectés par les changements dans leur entreprise, dont l'emploi est supprimé, qui sont contraints au départ sans avoir de projet professionnel précis sont des salariés licenciés pour motif personnel. Ils subissent la rupture et ont un risque élevé d'exclusion professionnelle. Il reste une zone intermédiaire qui se différencie de la précédente 


\section{Encadré 3}

\section{Les cinq cas observés de mobilisation d'un projet professionnel}

Poursuivre sa carrière: le salarié souhaite poursuivre sa carrière dans le même domaine d'activité et sensiblement dans les mêmes conditions d'emploi; la rupture du contrat de travail ne s'accompagne alors pas de rupture professionnelle; le salarié continue à exercer son activité dans le même domaine professionnel avec éventuellement un élargissement de ses responsabilités ou une promotion hiérarchique. II a simplement changé d'employeur sans difficultés particulières.

Trouver sa voie : le projet du salarié débouche sur la valorisation dans un nouvel emploi des compétences qu'il a acquises de sa propre initiative durant ses précédents emplois. Pour certains salariés, un des moteurs de leur rupture de contrat de travail est moins le contexte de restructuration de leur entreprise que leur projet professionnel propre et le fait qu'ils suivent depuis longtemps, en même temps que leur activité salariée, des formations professionnelles ou des études universitaires en accumulant des diplômes qu'ils ne peuvent valoriser dans leur emploi présent. Ils sont dans une situation où les formations suivies leur servent à «trouver leur voie ", l'emploi dans l'entreprise $\mathrm{COI}$ pouvant alors être interprété a posteriori comme un emploi d'attente.

Se reconvertir : le salarié envisage une reconversion professionnelle durant la phase, plus ou moins longue, qui s'étend entre le moment où il devient certain que le contrat de travail va être rompu et la date précise de la rupture. La reconversion, catégorie typique de rupture professionnelle, conduit à un changement de métier effectif deux ans plus tard grâce aux formations reçues.

Se réorienter: le salarié s'engage, dans le même laps de temps que précédemment, dans une rupture professionnelle sans avoir de projet de changement de métier. L'évolution peut alors porter sur les conditions d'exercice du métier en passant par exemple au statut d'indépendant ou bien en changeant de secteur d'activité ou de catégorie d'entreprise. Le caractère "choisi » de ce type de transition est moins net que dans les cas précédents même pour ceux qui prennent l'initiative de leur départ. La transition s'effectue sous forte contrainte et oblige le salarié à «se réorienter».

Subir la rupture: le salarié ne semble pas avoir de projet professionnel clair. Son entreprise le pousse le plus souvent à la rupture de son contrat de travail et il est, deux ans plus tard, toujours en situation difficile. Ces salariés qui «subissent la rupture" risquent l'exclusion professionnelle puisqu'ils ne retrouvent pas de travail.

parce que non seulement l'emploi n'est pas nécessairement supprimé et le départ peut être choisi par le salarié, mais surtout parce que le salarié dispose d'un projet professionnel qui consiste ou bien en la réactivation d'un projet ancien ou bien en la mobilisation rapide d'un nouveau projet. Toutes les modalités juridiques de rupture du contrat de travail y sont représentées et la transition professionnelle vise soit à une reconversion, soit à une réorientation de carrière plus ou moins contraintes.

\section{Cinq types de transition professionnelle}

Nous présentons les cinq types de transition professionnelle motivés par les objectifs qui viennent d'être présentés (poursuivre sa carrière; trouver sa voie; se reconvertir; se réorienter; subir la rupture) en les replaçant dans les trajectoires professionnelles des salariés et en faisant jouer les critères de différenciation présentés plus haut. Dans les deux premières transitions, les changements dans les entreprises sont mis à distance dans le discours du salarié qui, le plus souvent, a lui-même pris l'initiative de son départ en démissionnant. Dans les trois suivantes, les changements de l'entreprise sont au cœur des événements professionnels vécus : le départ peut aussi bien être imposé par l'entreprise que pris à l'initiative du salarié. La typologie des trajectoires n'est pas établie en fonction de profils sociaux typiques, que notre échantillon, non représentatif, ne nous permet pas, de fait, de déterminer précisément; les critères utilisés sont ici transversaux à des catégories professionnelles ou sociales d'où la difficulté, dans certains cas, à établir une correspondance avec des populations précises.

\section{Poursuivre sa carrière}

Le salarié prend l'initiative de son départ en démissionnant car il souhaite poursuivre sa carrière ou son projet professionnel dans le même domaine d'activité. Il est insatisfait de son emploi antérieur où ses compétences ne lui semblent pas reconnues; surtout, l'entreprise ne répond pas à ses souhaits d'évolution. En effet, le plus souvent, il est dans une phase ascendante de sa carrière et dans un contexte où il met à distance les changements dans son entreprise (voir tableau 1 en annexe). Dans certains cas, le salarié va jusqu'à considérer qu'il n'y a pas assez de changements dans l'entreprise ou que, s'il y en a, ils vont dans une mauvaise direction: Armand considère par exemple que son entreprise n'a pas suffisamment pris la mesure de la nouvelle activité qu'il développe et pour laquelle il a été recruté (la fidélisation sur Internet). Elle n'a pas transformé son organisation en conséquence: "On n'a pas les bons leviers de business », dit-il. Pierre regrette pour sa part l'orientation grand public prise par son magasin alors qu'il souhaite développer un secteur professionnel. Bien qu'ils soient issus d'origines sociales diverses - agriculteurs, ouvriers issus de l'immigration, cadres -, ces salariés forment un ensemble sociologiquement homogène : ce sont des hommes, jeunes, autour de la trentaine, diplômés 
du supérieur, en phase d'ascension professionnelle et de recherche de meilleures conditions d'emploi. Leur profil correspond à celui des salariés qui démissionnent dans l'enquête COI, modalité de rupture qui leur est de fait la plus fréquente. Outre leur sexe et leur âge, on remarque aussi qu'ils sont, pour la plupart, sans charge de famille et qu'ils travaillent souvent dans le secteur des services aux entreprises. Jonathan est représentatif de cette catégorie (voir encadré 4).

\section{Encadré 4
Jonathan, du développement au support
logiciel}

Jonathan vit seul. II a 32 ans en 2008, son père est agriculteur et sa mère au foyer. Titulaire depuis 1999 d'un diplôme d'ingénieur, il passe un an en Californie (Silicon Valley) où il obtient un master. II travaille alors comme «ingénieur en logiciel embarqué " dans une entreprise américaine de processeurs réseaux. Licencié en octobre 2001, il rentre en France. II est embauché sur profil (développeur pour des processeurs logiciels embarqués) dans une société de services informatiques et est rapidement envoyé sur une première mission de quinze mois qui est prolongée. II enchaîne ensuite avec une courte période d'intercontrat, puis une deuxième mission. Pendant ce temps, sa société, en forte croissance, achète d'autres sociétés et se restructure en pôles de spécialité.

À l'occasion de sa dernière mission, il effectue en 2006 un voyage aux États-Unis et rencontre une entreprise américaine de télécommunication qui lui propose de faire du support en Europe. Cette proposition arrive au bon moment car il se pose des questions sur l'évolution de sa carrière. II sait que, dans sa SSII (1), il n'obtiendra pas d'évolution, ni professionnelle, ni salariale. II prend conseil auprès de son ancien employeur américain et auprès d'une avocate, amie de ses parents, sur la clause de non-concurrence de son contrat de travail et sur les spécificités des contrats de travail américains. II démissionne et signe son nouveau contrat. Dans son nouvel emploi "Field application engineer", Jonathan est chargé du support pré et postvente. II dépend directement du directeur commercial de la zone Europe à Boston. La ligne hiérarchique est très plate et il apprécie de pouvoir rencontrer fréquemment son directeur. Grâce à un équipement en moyens de communication, il travaille depuis son domicile à Paris et effectue de fréquents déplacements aux États-Unis et en Europe. La seule pression à laquelle il s'estime soumis vient des échéances des clients. Les seuls désagréments qu'il signale sont l'absence de contacts directs et quotidiens avec des collègues.

(1) Société de services en ingénierie informatique.

Jonathan se caractérise par sa volonté de faire carrière dans son domaine professionnel. Il ne supporte pas de se sentir bloqué. Il sait prendre l'initiative de son évolution et gérer son contrat de travail dans une relation plus contractuelle que subordonnée. Il fait preuve d'une parfaite autonomie dans son organisation et dans la gestion de son temps de travail. Son intérêt pour son travail et le fait que son activité se traduise par une rémunération adéquate le motivent. Intégrant les contraintes marchandes de son secteur, il agit en fonction d'indicateurs de marge financière. Il apprécie d'avoir des contacts directs avec la direction de son entreprise car, d'une part, il ne souhaite pas avoir affaire à une structure hiérarchique qu'il juge pesante et démotivante et, d'autre part, il souhaite être au plus près des orientations stratégiques de son entreprise. En revanche, il tient les syndicats en piètre estime et ne considère absolument pas qu'ils puissent constituer pour lui une ressource d'action (7). Sur le plan relationnel, Jonathan considère que l'entretien d'un réseau professionnel fait pleinement partie de son activité et est une composante de son identité professionnelle. Il contribue à sa réputation et joue un rôle important pour trouver un nouvel emploi. En effet, ce sont les compétences du travailleur - et leur mise en avant via des réseaux - qui conditionnent sa mobilité et constituent sa valeur sur un marché professionnel (EYraUd et al., 1990; MARSDEN, 1999).

\section{Trouver sa voie}

On trouve d'autres salariés, proches des précédents, pour lesquels l'emploi quitté était avant tout un emploi d'attente, qui a plus ou moins duré; pendant ce temps, le salarié a préparé son évolution afin de concrétiser son projet professionnel (voir tableau 2 en annexe). Anne, Oualid et Pauline suivent à leur propre initiative des formations qualifiantes parallèlement à leur emploi : un doctorat d'ethnomusicologie pour Anne qui espère un poste universitaire, un brevet de technicien supérieur (BTS) d'imprimerie pour Oualid dont le diplôme d'ingénieur algérien est d'autant moins reconnu qu'il travaille dans un environnement où il est exposé à une discrimination liée à son origine géographique; un BTS d'informatique pour Pauline qui souhaite devenir administratrice réseau. Sociologiquement, ces salariés forment un ensemble moins homogène que le précédent. Trois d'entre eux sont soit immigrés, soit issus de l'immigration et d'origine sociale ouvrière ou employée. Anne et Oualid occupent un emploi déqualifié par rapport à leurs compétences. Oualid y est contraint par son statut d'immigré récent; tel n'est pas le cas d'Anne qui est restée dix-sept ans dans un emploi de vendeuse tout en menant des études de haut niveau.

(7) De nombreux travailleurs, surtout ceux de haut niveau de qualification en France, assimilent l'action syndicale à la seule défense du personnel d'exécution (les ouvriers). Les résultats de l'exploitation de l'enquête Reponse 2004-2005 (Amossé, 2006) montrent d'ailleurs que les salariés français restent éloignés du dialogue social institutionnel. 
Pauline est représentative de ces salariés qui mettent à distance les changements de leur entreprise car ils attachent plus d'importance à leur propre projet professionnel (voir encadré 5). La rupture de leur contrat de travail leur permet de se diriger vers une activité professionnelle qu'ils jugent plus conforme à leurs aspirations.

\section{Encadré 5}

\section{Pauline, devenir administratrice réseau}

Pauline, 32 ans lors de l'entretien, est d'origine camerounaise. Son père et sa mère sont employés, elle vit en couple avec un conjoint employé. Elle a été licenciée en $\mathbf{2 0 0 6}$ pour faute en raison d'un refus de service à un client. Elle travaillait depuis deux ans et demi dans une entreprise prestataire de services informatiques de réservation de voyages en tant que technicienne support pour les clients d'une grande compagnie aérienne. Cette grande compagnie s'est séparée de la société de services où travaille Pauline et a revendu les parts correspondantes. Une partie des salariés de la compagnie a quitté la société de services. Cette restructuration n'est cependant pas à l'origine de la rupture du contrat de travail de Pauline : "L'entreprise elle-même ne me posait pas de problème. » Son emploi de technicienne support était un emploi d'attente destiné à lui apporter les compétences nécessaires pour réaliser son projet de devenir administrateur réseau: "J'avais pris ce poste parce que de toute façon il fallait bien que je vive. [...] À l'époque, les boîtes d'intérim disaient qu'il fallait commencer par le support pour pouvoir atteindre un jour le poste d'administrateur réseau. " Tout en occupant cet emploi, elle suit des cours par correspondance et obtient un BTS d'informatique en candidate libre. La qualification de son licenciement (faute professionnelle) ne la touche pas; bien au contraire, elle est plutôt soulagée car elle s'est rendu compte qu'elle n'apprenait rien dans son poste de technicienne et qu'il ne lui permettrait pas de devenir informaticienne: "Je voulais vraiment évoluer.» Elle profite de sa période de chômage pour démarrer un master spécialisé d'informatique à l'Institut national des télécommunications (INT). La période d'instabilité qui suit son licenciement la rapproche du métier espéré, qu'elle finit par exercer après avoir trouvé un poste grâce à une ancienne relation de travail. Elle devient salariée d'une SSII qui l'envoie en mission dans une grande entreprise agroalimentaire en tant qu'administratrice réseau. Elle s'occupe de tout ce qui concerne le serveur et les postes des utilisateurs. Elle est devenue cadre et son emploi la satisfait pleinement.

Comme nous venons de le voir, de nombreux salariés semblent supporter sans dommage le contexte de restructuration de leur entreprise (le plus souvent une fusion / acquisition et une réorganisation interne). Ce n'est toutefois pas toujours le cas. D'autres salariés placent en effet les changements dans leur entreprise au cœur de leur transition professionnelle. Parmi eux, certains partent de leur propre initiative; d'autres n'ont pas le choix, un licenciement (ou une démission) leur étant imposé. Leur transition peut alors se traduire par une reconversion, une réorientation ou une rupture subie.

\section{Se reconvertir}

Les cas de reconversion professionnelle effective ou en préparation (changement de métier) sont nombreux parmi les salariés rencontrés (voir tableau 3 en annexe et encadrés 6 et 7). Leur départ prend la forme d'un licenciement : quand il est subi, leur emploi est supprimé après leur départ; lorsqu'il est choisi, leur emploi est maintenu. Des actions de formation accompagnent la reconversion. Certains salariés ont pu bénéficier de l'encadrement d'une cellule de conversion, d'autres non. Les projets sont souvent anciens même s'ils ne se sont jamais concrétisés. Les trois congés de conversion dans le cadre de plans sociaux négociés collectivement ont débouché sur des formations de longue durée, bien conduites et encadrées dans les cas de Claire et de Yann, sans encadrement dans le cas de Séverine. Certains projets de reconversion accompagnés de formation peuvent aussi être conduits en dehors de tout encadrement institutionnel. C'est le cas d'Édouard, d'autant qu'il n'a pas bénéficié d'un licenciement économique. Différence de taille avec les trois cas précédents: le salarié finance alors entièrement sa formation. Ou bien il vit seul et restreint son train de vie; ou bien il a un foyer, et sa reconversion devient un projet familial.

Dans cette catégorie, les salariés sont plus âgés que les précédents; ce sont aussi bien des hommes que des femmes qui ne se distinguent ni par leur niveau de diplôme, ni par leur catégorie professionnelle : ils sont cadres, employés ou ouvriers. Leurs origines sociales sont également diverses - agriculteur, issu de l'immigration, employé, cadre -, avec une prédominance de la catégorie cadre, pour cinq d'entre eux.

Tous ces salariés concernés par une reconversion professionnelle dans un contexte où ils s'estiment directement touchés par des changements intervenus dans leur entreprise ont fait l'objet d'un licenciement. Cependant, les conditions du licenciement comme l'initiative de la décision de départ diffèrent: au sein des licenciés pour motif économique, il existe une césure entre les cadres qui bénéficient du choix des modalités de leur départ et le personnel d'exécution pour lequel tout est imposé (Claire et Yann versus Séverine). Les LME se déroulent dans un contexte de restructurations d'entreprises de grande taille, souvent accompagnées d'un plan social. Les licenciés pour motif personnel en revanche se voient tous imposer le départ, quelle que soit leur catégorie professionnelle. Les deux LMP (Christine et Édouard) ont lieu dans un contexte de conflits internes où l'entreprise cherche à se séparer des anciens et à renouveler les équipes de travail. 


\section{Encadré 6}

\section{Édouard, de la SSII à l'orthophonie}

Édouard (34 ans, ingénieur informaticien et sept ans d'ancienneté dans une grosse SSII) se caractérise par son rejet des conditions de travail dans les grandes entreprises, en restructuration quasi permanente. II a le sentiment d'être ballotté sans avoir de prise sur son travail, ce qui motive sa reconversion vers un travail non subordonné, dans des activités d'interaction et de soin en face à face. La SSII qui l'emploie est en effet un groupe qui rachète, revend et scinde en permanence des sociétés. Cette instabilité engendre de nombreux mouvements internes de personnel. Édouard change cinq fois de responsable en sept ans et finit par se retrouver en décalage avec sa structure d'appartenance(1). Durant sa dernière période d'intercontrat (huit mois avant son départ), son employeur souhaite le faire démissionner puis tente de le licencier. Édouard parvient à négocier un «protocole d'accord transactionnel de licenciement». Durant cette période, aidé en cela par son réseau social, il se rend compte que l'informatique n'est pas une activité qui lui plaît: «J'ai beaucoup d'amis qui sont dans le secteur social, médicosocial. [...] J'ai vu ce qui me correspondait, ce que j'avais envie de faire et ça a été assez rapide à décider. " II opère ce qu'il appelle "un choix de vie", une reconversion vers des activités de soins à la personne. La capacité à renoncer à un salaire relativement élevé, qui est aussi un marqueur social de réussite, pour privilégier à la fois son propre épanouissement et le bien-être d'autrui est une des caractéristiques fondamentales d'Édouard. Sa famille, qui approuve son choix, le soutient à la fois moralement et financièrement, et consent à des sacrifices : Édouard réussit en effet le concours d'entrée de l'École d'orthophonie dont il suit la formation pendant quatre ans, à partir d'octobre 2007, alors qu'il ne perçoit plus aucune indemnité, ce qui sera le cas jusqu'en 2011. Sa reconversion est donc un processus collectif qui engage sa famille entière dans un autre projet de vie, décidé collectivement.

(1) Bien que le but des réorganisations ait été l'adéquation entre les compétences des ingénieurs et le département et la branche où ils sont affectés.

Entre le moment où il devient certain que le contrat de travail va être rompu et la date précise de la rupture, ces salariés élaborent un projet de reconversion qui peut avoir plusieurs origines. Pour certains d'entre eux, leur motivation est le désir de fuir les grandes entreprises où ils ont le sentiment de ne pas avoir de prise sur ce qui leur arrive, où les décisions qui ont un impact sur leur activité tombent de haut, de loin... Ces salariés, tels Claire ou Édouard, manifestent des difficultés à gérer leur place dans une grande organisation en restructuration et critiquent leur entreprise. Le contexte de changements permanents qu'ils vivent les pousse à se reconvertir vers des métiers qui leur semblent offrir davantage de satisfaction personnelle. Pour d'autres, leur motivation première est la réactivation d'un projet professionnel ancien, jamais concrétisé, et qui trouve dans le caractère inexorable de la rupture du contrat de travail, les moyens de sa réalisation (Christine, Séverine).

\section{Encadré 7}

\section{Séverine, de contrôleuse-qualité fabrication à aide-soignante}

Séverine (36 ans, contrôleuse-qualité fabrication et huit ans d'ancienneté, LME) vit en couple et est mère de deux enfants. Elle a toujours vécu à la campagne, ses parents et son mari sont agriculteurs. Après son baccalauréat, elle trouve rapidement en 1998 un premier emploi dans une entreprise soustraitante en logistique et transport de marchandises, proche de son domicile, qui embauche les jeunes de la région. Contrôleuse-qualité pour les marchandises exportées, elle a la responsabilité du contenu des colis et de leur conditionnement. Déjà à cette époque, elle rêve de devenir infirmière. Depuis plusieurs années, son entreprise est en difficulté économique, Séverine le sait mais n'envisage pas de partir car les possibilités de travail dans son bassin d'emploi sont limitées. Comme elle le dit, elle attend que le bateau coule. Le naufrage arrive en 2005 sous la forme de la perte d'un contrat avec la Chine qui entraîne une baisse importante d'activité. Durant toute l'année 2006, les salariés sont soumis à une grande flexibilité des horaires et sont maintenus en emploi alors qu'il n'y a plus de travail, tandis que direction et syndicats se consacrent à la mise au point des plans sociaux. Les salariés, tout au moins ceux du même niveau que celui de Séverine, ne sont pas tenus au courant des tractations et de l'avenir que leur réserve leur entreprise $^{(1)}$, ce qui provoque un climat de stress. Séverine se voit proposer deux mutations dans le sud de la France, inenvisageables car elle ne souhaite pas devenir "célibataire géographique» (VIGNAL, 2006b). Elle reçoit ensuite, sans rencontre préalable avec sa direction(2), une lettre de licenciement et bénéficie d'une année de formation dans le cadre de la cellule de reclassement. Séverine opte pour une formation d'aide-soignante. Elle a anticipé sa reconversion vers une activité à laquelle elle pensait depuis longtemps en repérant les différentes formations possibles avant même de recevoir sa lettre de licenciement: "J'étais à l'école, à la préparation du concours, je n'étais pas encore licenciée. » Elle ne peut toutefois qu'approcher son métier rêvé et non pas l'atteindre. Elle a, en effet, été licenciée en tant qu'opératrice de production, qualification inférieure à celle du métier qu'elle a réellement exercé. Cette déqualification ne lui ouvre droit qu'à un an de reclassement et surtout, ne lui permet pas de bénéficier du second plan social plus avantageux qui lui aurait permis de préparer le diplôme d'infirmière. Après un an d'école, elle est aussitôt embauchée comme aide-soignante contractuelle dans un hôpital proche de chez elle.

(1) Cette pratique est courante de la part des directions d'entreprises (LinHART et al., 2009).

(2) Cette rencontre est une obligation légale dans le cas des licenciements économiques. 


\section{Se réorienter}

Proche de la catégorie précédente en termes de composition sociologique, un autre ensemble se détache qui se caractérise par le fait que les salariés sont amenés à réorienter relativement rapidement leur carrière, sans action de formation de longue durée, entre le moment où ils acquièrent la certitude qu'ils ne pourront pas rester dans leur entreprise et le moment où leur contrat de travail est rompu. Cette période est souvent marquée par un mauvais climat relationnel et des conflits internes. La réorientation se traduit par un changement de statut d'emploi ou de caractéristique d'entreprise (passage au statut d'indépendant, nouveau poste au sein de la fonction publique, d'une structure associative ou d'une entreprise plus petite, etc.) (voir tableau 4 en annexe). Même si le départ peut être choisi ou subi et l'emploi supprimé ou maintenu, cette forme de rupture professionnelle consiste en une réorientation vers des modalités d'exercice de l'activité professionnelle que les salariés présentent comme moins exposées aux changements.

Les trois démissions (Gabriel, Jean-Charles, Sylvie) concernent des salariés qui n'étaient pas menacés dans leur emploi mais dont l'entreprise manifeste des difficultés d'organisation qui s'expriment notamment par des conflits internes. Là où les catégories socioprofessionnelles jouent entre ces salariés, c'est au moment de la négociation de leur départ avec leur employeur (voir supra, «Les transitions professionnelles des salariés»). Les cadres de haut niveau de diplôme s'autorisent et parviennent à négocier des conditions beaucoup plus favorables que les ouvriers et les employés (Joseph). Le LMP (Joseph) a lieu dans un contexte de dissensions internes où l'entreprise cherche à renouveler les équipes et à se séparer des anciens. Le cas de Joseph est un peu particulier: il a été licencié pour refus de mutation mais, son entreprise étant visiblement en tort, il a obtenu un an de coaching pour affiner son projet, ce qui lui a permis ensuite de créer son entreprise et de devenir indépendant. Les LME rassemblent deux situations tout à fait distinctes. Il y a deux LME individuels d'un côté (Adélaïde et Étienne) : Adélaïde est mise à l'écart à son retour de congé de maternité tandis qu'Étienne est en conflit avec son employeur qui est son ancien associé. De l'autre, un LME collectif (Myriam) qui présente la situation suivante : l'employeur a prévu les modalités de sortie de ses employés au moment de son départ en retraite et de la cession de son entreprise à une plus grande.

Les circonstances de départ de l'entreprise sont donc diverses. Dans un premier cas, l'emploi du salarié n'est pas menacé; néanmoins les conditions de travail sont dégradées en raison de l'intensification de la charge de travail due à une mauvaise organisation (Jean-Charles) ou bien d'un déménagement imposé suite au rachat de l'entreprise (Myriam). Il arrive également que le salarié travaille dans un climat de relations sociales peu sereines et qui se sont considérablement dégradées dans les derniers mois suite à une ou plusieurs réorganisations internes. Il peut d'autant moins ajourner son départ que son employeur veut se débarrasser de lui (Adélaïde, Joseph; voir encadré 8). D'autres salariés saisissent une opportunité qui semble se présenter par un bienheureux hasard (Sylvie, Gabriel). Gabriel (voir encadré 9) considère avoir bénéficié d'une occasion, arrivée au bon moment grâce à ses relations personnelles, qui lui a permis de se mettre à l'abri des turbulences. Ce qui est présenté comme un hasard joue un rôle, «précisément parce qu'il s'inscrit dans une configuration particulière qui est celle dans laquelle se trouve l'individu à un instant précis. Il ne se déclenche que parce qu'il y a une direction préexistante en filigrane» (NeGRoni, 2005, p. 317).

\section{Encadré 8 \\ Joseph, la mobilisation rapide de ressources pour devenir indépendant}

Joseph (56 ans, chargé d'affaires et cinq ans d'ancienneté dans une grande entreprise de traitement des eaux, LMP) n'a pas anticipé sa rupture professionnelle. II n'a pas non plus pris l'initiative de son départ. L'année précédente, son entreprise subit une série de restructurations : elle est rachetée par un LBO (1), le siège déménage et son supérieur hiérarchique direct est remplacé. II a été mis devant le fait accompli, dans une situation psychologiquement difficile à supporter car son entreprise voulait se débarrasser de lui : "ll y a une pression telle qui est faite sur des gens comme nous. Au bout d'un moment, ils provoquent une situation de façon tellement tendue, sans aucun respect de la personne. " Joseph mobilise alors des ressources qui lui permettent de réagir rapidement et de trouver une nouvelle orientation de carrière. Il présente sa rupture professionnelle comme une décision prise relativement rapidement. Le refus de mutation invoqué par l'entreprise ne tient pas du fait du contenu de son contrat de travail. II est alors en bonne position pour négocier un licenciement à l'amiable avec l'octroi d'un coaching pendant un an. Deux éléments lui ont permis de trouver les ressources nécessaires pour revendiquer ses droits et se lancer dans la création d'entreprise. II a été révolté par le traitement que son entreprise lui a réservé. Mais surtout, il dispose des capitaux nécessaires à sa réorientation : haut niveau de formation (doctorat), expérience professionnelle solide (trente ans), bonne rémunération, réseau professionnel large.

(1) Leverage Buy Out : montage de rachat d'entreprise par recours à l'endettement bancaire. Une holding est créée pour acheter des parts de la société-cible grâce à un emprunt bancaire. Les charges financières de la dette sont payées par les dividendes de la cible. 


\section{Encadré 9}

\section{Gabriel, la saisie d'une opportunité qui lui permet de se mettre à l'abri}

Gabriel (42 ans, six ans d'ancienneté, magasinier au service carrosserie d'une concession automobile) a saisi une opportunité d'emploi. Après un certificat d'aptitude professionnelle (CAP), il a commencé à travailler à 19 ans dans une première concession automobile. En 2006, il démissionne d'une autre concession automobile du même constructeur où il est resté six ans, toujours en tant que magasinier. II aspire à une promotion professionnelle de chef de magasin pour laquelle il s'estime compétent. Sa concession automobile appartient à un groupe qui rachète d'autres constructeurs. Ces changements provoquent chez les salariés de l'inquiétude pour l'avenir. La charge de travail s'alourdit, ce qui conduit à des frictions entre collègues. Gabriel explique que l'intensification du travail provient des remplacements qu'il faut effectuer au pied levé en plus de son propre travail. Au manque de personnel s'ajoute une organisation peu rationnelle de l'espace : le téléphone pour les commandes est à l'étage et l'atelier pour les réparations au rez-dechaussée. La pénibilité est non seulement mentale mais aussi physique car les pièces de carrosserie sont lourdes à transporter. Le discours de Gabriel révèle de sérieux dysfonctionnements ergonomiques. Il est critique vis-à-vis des actions commerciales mises en place et des incitations à la vente qui sont imposées aux salariés : une prime en fonction du nombre de pièces détachées vendues. II ne se sent pas prêt à pousser à la vente coûte que coûte. C'est alors qu'une occasion se présente : il apprend par un ancien collègue que celui-ci libère son poste dans une Chambre des métiers. Son insatisfaction par rapport à son absence de promotion, le climat général d'inquiétude et la dégradation tout à fait concrète de ses conditions de travail l'ont rendu plus ouvert aux opportunités et lui permettent de prendre l'initiative de son départ. II démissionne pour ce poste d'agent d'entretien et de sécurité. Au bout d'un an, il est titularisé comme fonctionnaire.

\section{Subir la rupture}

D'autres salariés, en l'absence d'un projet professionnel précis, ne parviennent pas à mobiliser les ressources qui leur permettraient de se réorienter et de trouver un nouvel emploi. Ils restent sans solution professionnelle, comme Antonio, Catherine et Lise, deux ans après la rupture de leur contrat de travail. Des événements de santé interfèrent en plus parfois. Tous trois ont fait l'objet d'une modalité particulière de licenciement : un LMP alors que la raison économique pouvait s'appliquer (voir le tableau 5 en annexe et les cas de Catherine et d'Antonio en encadrés 10 et 11). La raison invoquée pour deux d'entre eux - l'inaptitude pour l'un, l'insuffisance professionnelle pour l'autre - cache des handicaps physiques qui obèrent un recrutement ultérieur. Pour la troisième, son domicile dans un bassin d'emploi peu prospère et une relation d'emploi atypique qui l'a isolée sont autant d'éléments qui s'associent pour réduire ses chances de recrutement.

Ces salariés se distinguent par une homogénéité sociale qui n'apparaît pas dans leurs niveaux d'études variés (du brevet d'études professionnelles [BEP] à la maîtrise universitaire), mais plutôt à travers leur origine sociale populaire (agriculteurs, issus de l'immigration, petits commerçants, ouvriers) ou à travers la catégorie professionnelle de leur conjoint (agriculteur, ouvrier, employé).

\section{Encadré 10 \\ Catherine, le piège d'une relation d'emploi atypique qui isole}

Catherine est dans une situation tout à fait paradoxale: elle a demandé à être licenciée mais ne souhaitait pas partir. C'est seulement en raison de la spécificité de son mode de rémunération (à la pièce) que son initiative trouve sa justification: "J'aurais pu rester sans être licenciée, sans démissionner. Je restais employée de la société sans avoir de travail. Donc ça ne rime à rien! » Mère de famille de 48 ans d'origine ouvrière, son conjoint est employé. Après un BEP de secrétariat en 1979, elle enchaîne des missions d'intérim dans différentes entreprises jusqu'à la fin 1983. À la naissance de son troisième enfant, elle arrête de travailler jusqu'en 1995. À cette date, une de ses amies, couturière à domicile, tombe gravement malade et cesse son activité. Catherine décide de prendre la suite et se fait embaucher comme couturière à domicile à la tâche pour la confection de drapeaux par une petite entreprise familiale qui réalise en sous-traitance des bâches de camion et des pavillons pour des groupes automobiles français ${ }^{(1)}$. Catherine finance son équipement : machine à coudre industrielle, véhicule pour transporter les cartons de drapeau, etc. Elle aménage elle-même son domicile. Elle est entièrement libre de ses horaires, très flexibles. Son travail est soumis au rythme des commandes et peut être très intense. Ses enfants et son mari sont mis à contribution : ils l'aident à plier les drapeaux. Son salaire en revanche est fixe, au niveau du salaire minimum, quel que soit le nombre d'heures travaillées. À partir des années 2000 , les commandes se raréfient. En 2004, l'entreprise essaie de gagner en productivité en intensifiant le rendement: cinq minutes par drapeau au lieu de sept mais, comme cela se révèle impossible à réaliser, l'entreprise décide en $\mathbf{2 0 0 6}$ de délocaliser la production des drapeaux en Chine. La conséquence immédiate est que Catherine n'a plus de travail; son employeur souhaite la garder en cas de surcroît d'activité mais ne la rémunère pas. Elle se décide à demander un licenciement. Elle ignore tout de ses droits et se fait accompagner par un représentant syndical qui ne semble guère plus informé. Non seulement elle n'obtient rien mais depuis, son patron refuse de la réembaucher sur d'autres postes pour lesquels il recrute. Elle est toujours sans emploi en 2009. Sa relation d'emploi, tout à fait particulière 
et archaïque, l'a isolée du collectif de travail de l'entreprise. Intervenant comme un prestataire de services extérieur, elle ignore tout du fonctionnement de son entreprise et ne peut se constituer un réseau professionnel sur lequel s'appuyer en cas de difficultés. Son seul soutien reste ses ressources familiales. Si l'on observe sa trajectoire professionnelle sur le long terme, on s'aperçoit que son ancrage identitaire principal est son rôle de mère de famille: "Quand j'ai commencé, j'avais quatre enfants et pour moi, c'était l'idéal de pouvoir travailler à la maison, afin d'être disponible pour les enfants. »

(1) Pour comprendre le contexte, il faut savoir que Catherine habite le Nord de la France, une région durement touchée par la crise depuis les années 1970. Son entreprise ne semble cependant pas dépourvue d'avenir économique. Elle a développé son identité à partir de son savoir-faire centenaire et diversifié ses débouchés tout en utilisant les avantages de la mondialisation des échanges qui lui permet de délocaliser en Chine les segments les plus simples de sa production.

\section{Encadré 11}

\section{Antonio, la stigmatisation du handicap}

Antonio, 34 ans, est d'origine portugaise. Titulaire d'une maîtrise en chimie organique, il a été recruté en 1999 par une petite société de services informatiques. II s'agit de son premier emploi. Il est formé au langage Cobol puis envoyé sur une première mission. Malheureusement, il tombe malade et doit s'absenter deux années complètes. À son retour, handicapé par sa maladie, il doit réduire son temps de travail et passe à $80 \%$. Son entreprise, qui ne souhaite pas se séparer de lui, le forme à un nouveau langage, Java, et lui trouve une mission à mi-temps. Entretemps, la SSII est rachetée une première fois par un groupe belge. Puis, en 2006, le président directeur général créateur de la SSII part à la retraite et l'entreprise est rachetée par un groupe américain. Ce rachat a deux conséquences immédiates: la mise à l'écart des anciennes équipes et le renforcement des objectifs de rentabilité. L'entreprise souhaite qu'Antonio démissionne et le place dans une situation psychologiquement difficile à supporter pour se débarrasser de lui : "Sentir qu'on vous entraîne vers la sortie, qu'on essaie de vous pousser, tout en vous faisant penser que c'est de votre plein gré. " II réussit à négocier un LMP qui lui permettra de percevoir des indemnités de chômage. Le motif officiel du licenciement (insuffisance professionnelle) le touche d'autant plus profondément qu'il n'en a jamais fait preuve. Ce motif, non seulement fallacieux mais stigmatisant, vient renforcer l'effet de son handicap. Le licenciement est le point de départ d'une période difficile : non seulement il arrive en fin de droits Assedic en 2008 mais, de plus, ses projets d'orientation professionnelle ne sont pas très précis.
Sans projet d'avenir, Catherine et Antonio se trouvent particulièrement déqualifiés dans le monde professionnel actuel: "Celui qui, n'ayant pas de projet, n'explore plus les réseaux, est menacé d'exclusion, c'est-à-dire en effet de mort dans un univers réticulaire. Il risque de ne plus trouver à s'insérer dans des projets et de ne plus exister» (Boltanski, Chiapello, 1999, p. 168). Les règles du jeu social sont en effet implacables, notamment pour les plus faibles : «C'est aux individus de fixer leurs propres fins à travers des projets et contrats» (Astier, Duvoux, 2006). Ce profil de trajectoire se trouve aussi dans l'exploitation statistique de l'enquête $F Q P 2003$ : «Le profil associé aux retraits et exclusions du marché du travail est celui de salariés avec peu de ressources à faire valoir pour retrouver un emploi... dont les emplois se situaient à la périphérie du monde salarié (travail à domicile, travail à temps partiel)» (AмOSsé et al., 2011, p. 93).

$$
\begin{array}{ll} 
& * \\
& \\
* & *
\end{array}
$$

L'objectif de cet article était de mettre au jour les transitions professionnelles des salariés qui voient leur contrat de travail volontairement ou involontairement rompu dans un contexte de changements organisationnels et technologiques des entreprises. Quels sont les effets des changements dans les entreprises sur les ruptures de contrat de travail et les transitions professionnelles? Pour répondre à cette question nous avons exploité l'enquête COI 2006 auprès d'un échantillon aléatoire de salariés dont une sélection a été réinterrogée lors d'un entretien réalisé deux ans plus tard.

La problématique développée considère les transitions professionnelles des salariés comme des interactions entre des déterminants structurels et les stratégies professionnelles des salariés. L'éventail des transitions est large entre ceux qui se saisissent du changement et de la rupture pour développer un projet professionnel, ceux qui provoquent la rupture pour se reconvertir et ceux qui subissent les événements sans sembler avoir de prise sur eux.

Au-delà d'une approche dichotomique qui sépare les démissions des licenciements et les transitions choisies des transitions subies, il existe une graduation qui va de la modalité de rupture du contrat de travail la plus choisie à la modalité la plus contrainte. Sans remettre en cause le résultat selon lequel, «les démissions sont surreprésentées dans les trajectoires favorables de promotion alors que les licenciements sont plus fréquemment associés aux trajectoires de retrait-exclusion ou d'emploi-chômage long» (Amossé et al., 2011, p. 98), ce travail met au jour une zone peu explorée où le traitement quantitatif est difficile dans la mesure où les catégories usuelles y sont inopérantes: toutes les modalités 
de rupture du contrat de travail sont représentées, l'emploi peut être ou non supprimé par l'entreprise, la transition peut être ou non choisie par le salarié. Il invite à la construction de nouvelles catégories d'analyse du fonctionnement du marché du travail et des mobilités professionnelles qui rendent compte de leur double origine - les pratiques de gestion des ressources humaines propres aux différentes catégories d'entreprise et les logiques d'action plus ou moins contraintes des salariés.

\section{Bibliographie}

Amossé T. (2006), «Le dialogue social en entreprise : une intensification de l'activité institutionnelle, des salariés faiblement engagés », Premières informations. Premières synthèses, $\mathrm{n}^{\circ} 39.1$.

Amossé T., Perraudin C., Petit H. (2011), «Mobilité et segmentation du marché du travail: quel parcours professionnel après avoir perdu ou quitté son emploi?», Économie et statistique, $\mathrm{n}^{\circ}$ 450, pp. 79-105.

Astier I., Duvoux N. (2006), La société biographique: une injonction à vivre dignement, Paris, L'Harmattan.

Beaujolin-Bellet R., Lerais F., Paucard D. (2012), «Introduction. Les modes de gestion des restructurations : quoi de neuf?», Revue de l'Ires, n ${ }^{\circ}$ 2, pp. 3-28.

Behaghel L. (2003), «Insécurité de l'emploi : le rôle protecteur de l'ancienneté a-t-il baissé en France?», Économie et statistique, $\mathrm{n}^{\circ} 366$, pp. 3-29.

BERTON F. (2000), «Acquérir un diplôme en cours de vie active, modèle social dépassé ou nouvel outil de gestion de la mobilité sur le marché du travail?», L'orientation scolaire et professionnelle, vol. 29, $\mathrm{n}^{\circ}$ 2, pp. 355-376.

Berton F., Huiban J.-P., Nortier F. (2011), «Les carrières salariales des hommes et des femmes : quelle convergence sur longue période?», Travail et emploi, $\mathrm{n}^{\circ} 125$, pp. 9-25.

Berton F., Perez C. (2010), «Démissions et licenciements: quels liens avec les changements organisationnels et/ou technologiques des entreprises?», Les Cahiers du Lise, $\mathrm{n}^{\circ} 3$.

Bessin M., Bidart C., Grossetti M. (dir.) (2010), Bifurcations. Les sciences sociales face aux ruptures et à l'événement, Paris, La Découverte.

Blosseville J.-M., Clémenceau P., Grando J.-M. (1982), «Les modes sectoriels de gestion de la main-d'œuvre: essai de caractérisation des secteurs industriels», Collection Études, $\mathrm{n}^{\circ}$ 1, Marseille, Céreq.

Boltanski L., Chiapello E. (1999), Le nouvel esprit du capitalisme, Paris, Gallimard.

BouRdieu P. (1986), «L'illusion biographique», Actes de la recherche en sciences sociales, ${ }^{\circ} 62-63$, pp. 69-72.

BruYÈre M., Lizé L. (2010), «Emploi et sécurité des trajectoires professionnelles. La nature de l'emploi détermine la sécurité des parcours professionnels», Économie et statistique, n 431-432, pp. 95-113.
Castel R. (1995), Les métamorphoses de la question sociale : une chronique du salariat, Paris, Fayard.

Combes M., Lethielleux L. (2008), «Comment prédire et expliquer l'échec des changements organisationnels », Revue française de gestion, $\mathrm{n}^{\circ}$ 188-189, pp. 325-339.

Dayan J.-L., Desage G., Perraudin C., Valeyre A. (2008) «La pluralité des modèles d'organisation du travail, source de différenciation des relations de travail», in Amossé T., Bloch-London C., Wolff L., Les relations sociales en entreprise : un portrait à partir des enquêtes. Relations professionnelles et négociations d'entreprise, REPONSE 1992-1993, 1998-1999, 2004-2005, Paris, La Découverte, pp. 334-352.

Denave S. (2006), «Les conditions individuelles et collectives des ruptures professionnelles», Cahiers internationaux de sociologie, $\mathrm{n}^{\circ} 120$, pp. 85-110.

Dubar C. (2001), «La construction sociale de l'insertion professionnelle», Éducation et sociétés, n ${ }^{\circ}$, pp. 23-36.

Eyraud F., Marsden D., Silvestre J.-J. (1990), « Occupational and internal labour markets in Britain and France », International labour review, vol. 129, $\mathrm{n}^{\circ} 4$, pp. 501-517.

Gazier B., Petit H. (2007), «French labour market segmentation and French labour market policies since the seventies: connecting changes», Économies et sociétés, tome XLI, série «Socio-économie du travail», pp. 1027-1056.

Gollac M. (2005), «L'intensité du travail: formes et effets», Revue économique, vol. 56, n 2, pp. 195-216.

Grossetti M. (2006), «L'imprévisibilité dans les parcours sociaux», Cahiers internationaux de sociologie, ${ }^{\circ} 120$, pp. 5-28.

GuYonVARCH M. (2008), «La banalisation du licenciement dans les parcours professionnels: déstabilisation ou recomposition des identités au travail? (enquête) ", Terrains \& travaux, $\mathrm{n}^{\circ} 14$, pp. 149-170.

Hudson M. (2002), «Flexibility and the reorganisation of work », in Burchell B., Ladipo D., Wilkinson F. (ed.), Job insecurity and work intensification, London, New York, Routledge.

Kocoglu Y., Moatty F. (2010), «Les entreprises ont-elles changé d'organisation? Une mesure à partir des déclarations des dirigeants », Réseaux, $\mathrm{n}^{\circ} 162$, pp. 199-229. 
Linhart D., Rist B., Durand E. (2009), Perte d'emploi, perte de soi, Ramonville-Saint-Agne, Érès.

Marsden D. (1999), A theory of employment systems: micro-foundations of diversity, Oxford, New York, Oxford university press.

NEGRONI C. (2005), «La reconversion professionnelle volontaire: d'une bifurcation professionnelle à une bifurcation biographique», Cahiers internationaux de sociologie, $\mathrm{n}^{\circ} 119$, pp. 311-331.

Palpacuer F., Seignour A., Vercher C. (2007), Sorties de cadres: le licenciement pour motif personnel, instrument de gestion de la firme mondialisée, Paris, La Découverte.

Petit H. (2002), Fondements et dynamique de la segmentation $d u$ marché du travail: une analyse sur données françaises, Thèse de doctorat en économie, Université Paris-I.

Signoretto C., Valentin J. (2012), «Pratiques des employeurs en matière de licenciements : une analyse sur données d'entreprises », Documents de travail du centre d'économie de la Sorbonne, $\mathrm{n}^{\circ}$ 2012-49.

VignAL C. (2005a), «Injonctions à la mobilité, arbitrages résidentiels et délocalisation de l'emploi», Cahiers internationaux de sociologie, $\mathrm{n}^{\circ} 118, \mathrm{pp}$. 101-117.

Vignal C. (2005b), «Les espaces familiaux à l'épreuve de la délocalisation de l'emploi : ancrages et mobilités de salariés de l'industrie», Espaces et sociétés, n 120-121, pp. 179-197.

Vignal C. (2006), «Devenir "célibataire géographique”? Arbitrages conjugaux et familiaux suite à la délocalisation d'une usine», Cahiers du genre, n 41, pp. 139-157. 


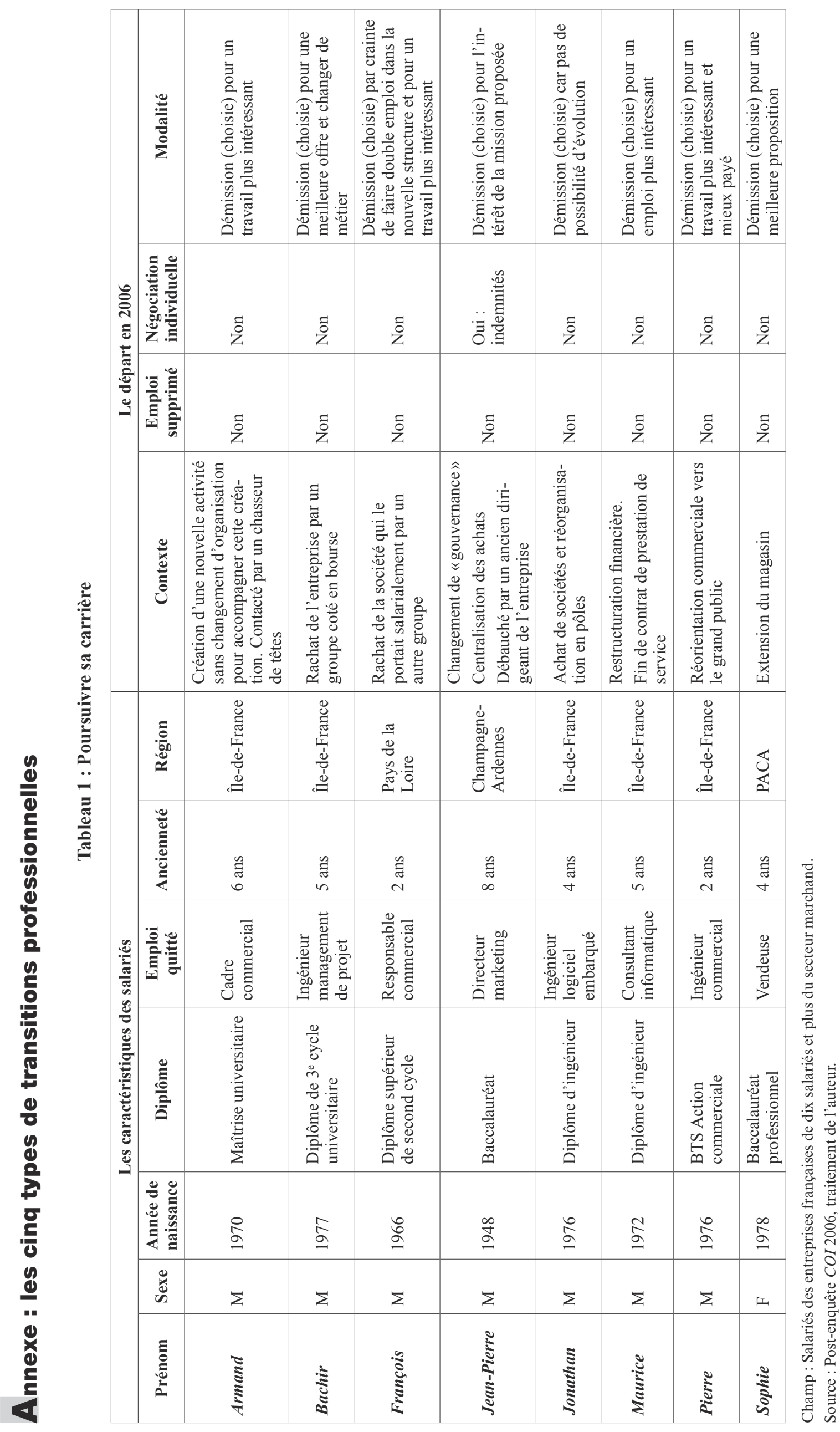

- 66 - Travail et Emploi $n^{\circ} 136$ 


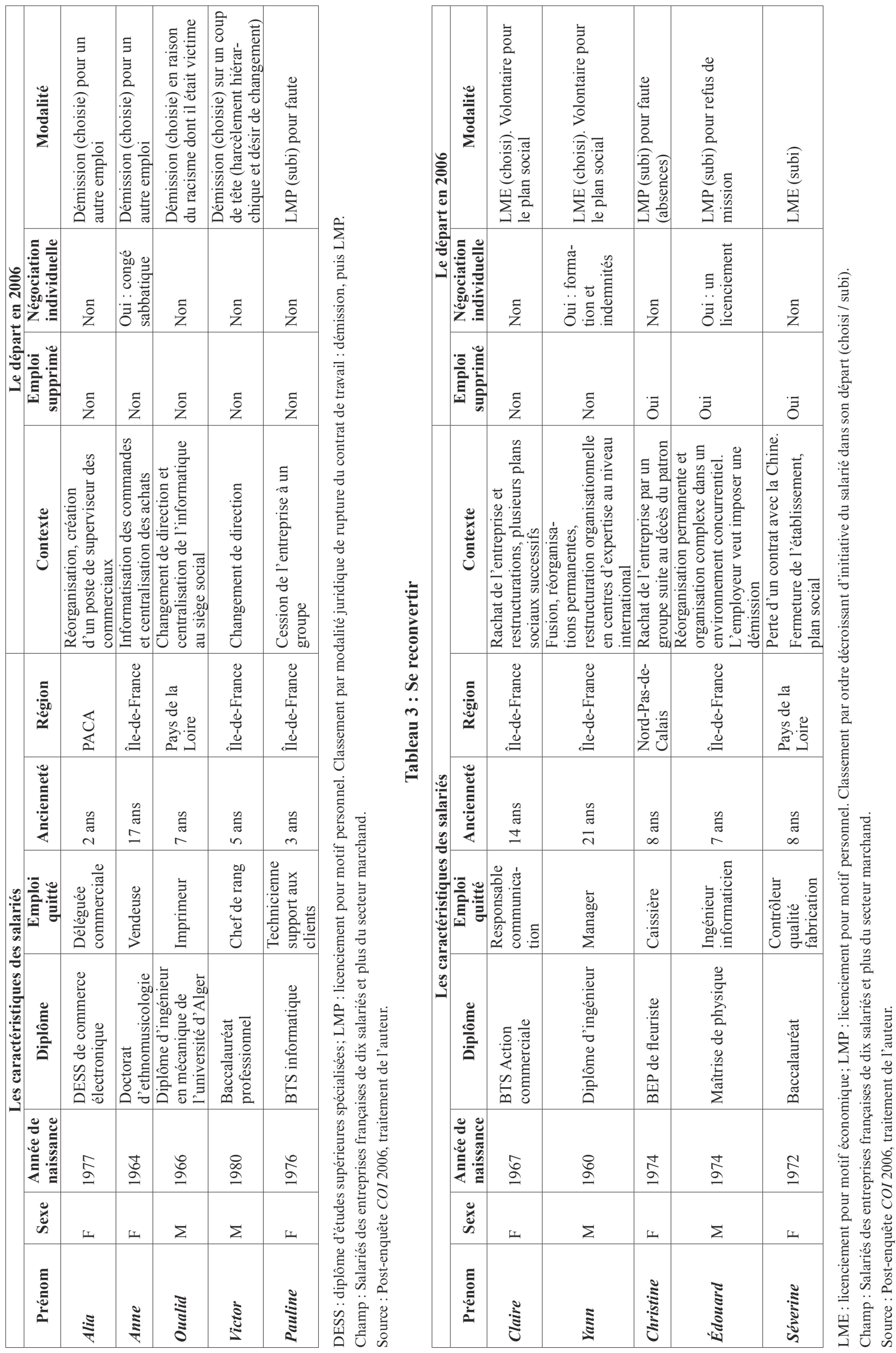




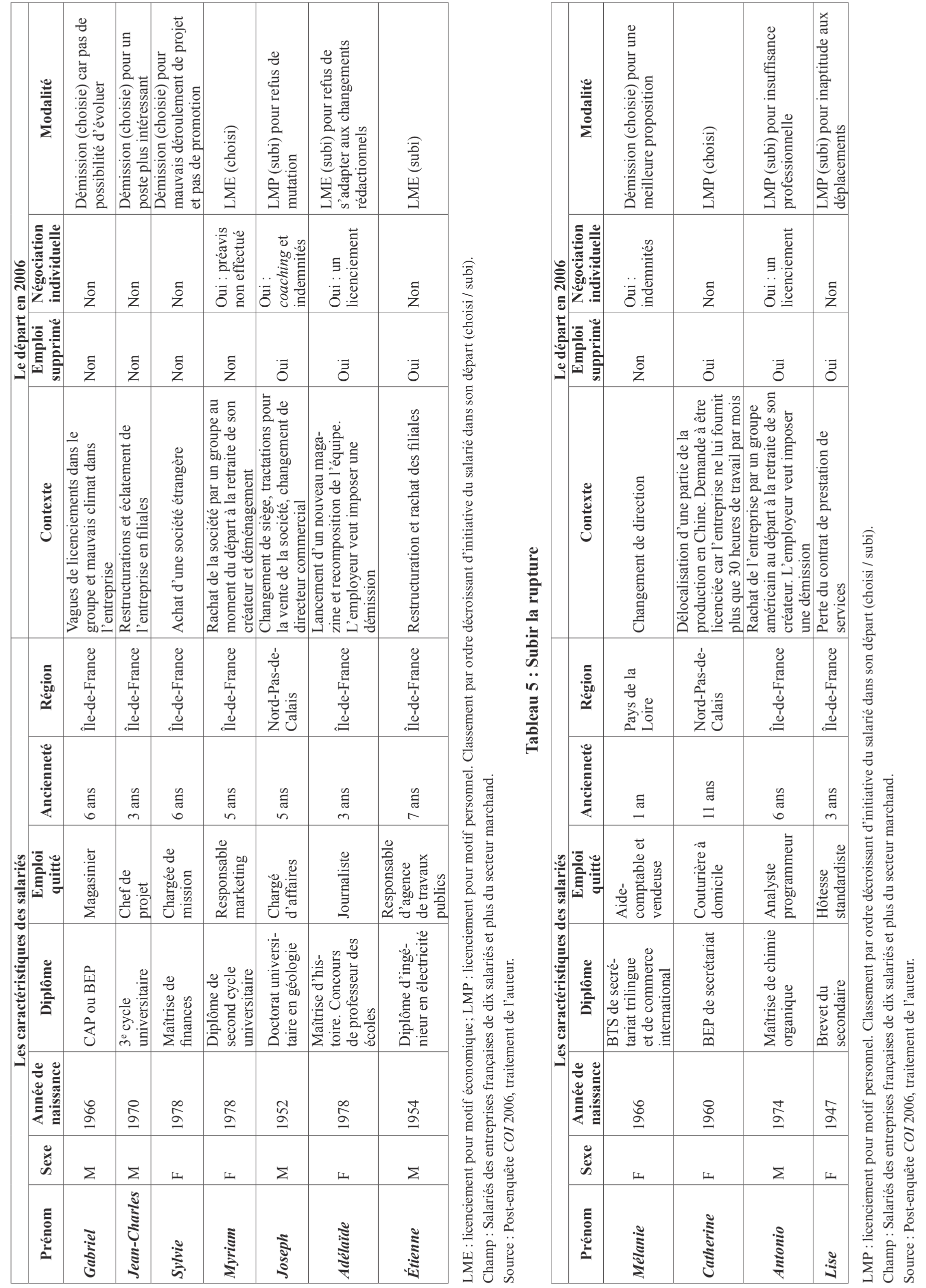

